\title{
Electrochemical immunosensor for simultaneous determination of interleukin-1 beta and tumor necrosis factor alpha in serum and saliva using dual screen printed electrodes modified with functionalized double-walled carbon nanotubes
}

\author{
E. Sánchez-Tirado, C. Salvo, A. González-Cortés, P. Yáñez-Sedeño*, \\ F. Langa ${ }^{1}$, J.M. Pingarrón
}

Department of Analytical Chemistry, Faculty of Chemistry, University Complutense of Madrid, Ciudad Universitaria s/n, 28040-Madrid, Spain; ${ }^{1}$ Instituto de Nanociencia, Nanotecnología y Materiales Moleculares (INAMOL), Universidad de Castilla-La Mancha, 45071-Toledo, Spain

*Corresponding author

Key words: interleukin-1 beta; tumor necrosis factor-alpha; electrochemical immunosensor; multiplex; double-walled carbon nanotubes; saliva. 


\begin{abstract}
Dual screen-printed carbon electrodes modified with 4-carboxyphenylfunctionalized double-walled carbon nanotubes (HOOC-Phe-DWCNTs/SPCEs) have been used as scaffolds for the preparation of electrochemical immunosensors for the simultaneous determination of the cytokines Interleukin-1 $(\mathrm{IL}-1 \beta)$ and factor necrosis tumor $\alpha(\mathrm{TNF}-\alpha)$. IL-1 $\beta$. Capture antibodies were immobilized onto HOOC-PheDWCNTs/ SPCEs in an oriented form making using the commercial polymeric coating Mix $\& G^{T M}$. Sandwich type immunoassays with amperometric signal amplification through the use of poly-HRP-streptavidin conjugates and $\mathrm{H}_{2} \mathrm{O}_{2}$ as HRP substrate and hydroquinone as redox mediator were implemented. Upon optimization of the experimental variables affecting the immunosensor performance, the dual immunosensor allows ranges of linearity extending between 0.5 and $100 \mathrm{pg} / \mathrm{mL}$ and from 1 to $200 \mathrm{pg} / \mathrm{mL}$ for IL-1 $\beta$ and TNF- $\alpha$, respectively, these ranges being adequate for the determination of the cytokines in clinical samples. The achieved limits of detection were $0.38 \mathrm{pg} / \mathrm{mL}(\mathrm{IL}-1 \beta)$ and $0.85 \mathrm{pg} / \mathrm{mL}(\mathrm{TNF}-\alpha)$. In addition, the dual immunosensor exhibits excellent reproducibility of the measurements, storage stability of the anti-IL-Phe-DWCNTs/SPCE and anti-TNF-Phe-DWCNTs/SPCE conjugates, and selectivity as well as negligible cross-talking. The dual immunosensor was applied to the simultaneous determination of IL- $1 \beta$ and TNF- $\alpha$ in human serum spiked at clinically relevant concentration levels and in real saliva samples.
\end{abstract}




\section{Introduction}

Cytokine detection is important for prognosis and diagnosis of different diseases related with inflammation, immunology, atherosclerosis and cancer [1]. However, monitoring of these proteins show some challenges related to their low concentration ranges in biological samples [2], which makes it difficult obtaining accurate and reliable analytical results. Moreover, as cytokines are known to work in concert to operate on the network of effectors [1] efficient analytical strategies should be implemented in the form of multiplexed detection [3]. It is well known that multianalyte approaches provide more information for each single sample and lead to faster and low-cost assays.

In this paper, we report the construction and analytical performance of an electrochemical platform for the simultaneous detection of two important cytokines: Interleukin-1 $\beta$ (IL-1 $\beta$ ) and factor necrosis tumor $\alpha$ (TNF- $\alpha)$. IL-1 $\beta$ is a proinflammatory cytokine produced by a variety of cells including pancreatic cells, adipocytes or macrophages. Similar to other cytokines, the determination of IL-1 $\beta$ is relevant for monitoring inflammatory and immune processes, but it is also significant in some specific cases. For instance, elevation of the IL-1 $\beta$ concentration is a typical reaction of the brain to acute injury and it can be found in a number of chronic neurodegenerative diseases such as Alzheimer [4]. In addition, high glucose concentrations induce the pancreatic expression of IL-1 $\beta$ leading to alteration of insulin secretion, as well as a decrease in cellular proliferation and apoptosis of pancreatic beta cells. On the other hand, the role of TNF- $\alpha$ is critical in many diseases including rheumatoid arthritis [5] or cancer [6].

Biological activities of IL-1 $\beta$ and TNF- $\alpha$, have been reported to be synergistic and overlapping [7]. For instance, both proteins exert an important inhibitory function on erythropoiesis blunting the endogenous production of EPO, and might play a role in 
the pathogenesis of anemia of chronic disorders or that of cancer patients which respond to recombinant EPO [8]. Moreover, increased IL-1 $\beta$ and TNF- $\alpha$ levels in vitreous humor and serum of patients with proliferative diabetic retinopathy has also been described [9]. Elevated levels of various cytokines, including IL-1 $\beta$ and TNF- $\alpha$, have also been identified in the circulation of traumatic brain injury (TBI) patients within hours of injury [10]. Interestingly, both proteins can be used as biomarkers to predict side effects of cancer therapy such as inflammatory oral mucositis [11]. In addition, determination of salivary contents of both cytokines in patients with leukoplakia and oral cancer has a great interest [12] and very recently they have also been considered as novel biomarkers for the detection of periodontal diseases [13].

Due to the normal levels of IL-1 $\beta$ and TNF- $\alpha$ in biological fluids are in the concentration range of few $\mathrm{pg} / \mathrm{mL}$, sufficient sensitivity is a requirement for the developed analytical methods. Therefore, enhancement in sensitivity has been a common issue for the previously reported electrochemical immunosensors. As Table 1 shows [14-30], the number of sensors described for TNF- $\alpha$ is relatively high whereas those for IL- $\beta 1$ are very scarce. Diverse electrode modifiers $[15,16]$, signal amplifiers $[16,17]$ and a variety of electrochemical techniques [18,19] have been employed to attain the required detection limits. Regarding the simultaneous determination of these cytokines, only three electrochemical immunoassay configurations have been reported (Table 1). An ultrasensitive microfluidic array was developed for TNF- $\alpha$, IL-6, IL-1 $\beta$, and C-reactive protein to assess oral mucositis risk in cancer patients. Magnetic beads were used to capture the analytes from serum and detected with a sensor array coated with gold nanoparticles and antibodies [11]. A fully integrated BioMEMS was also developed to simultaneously detect various interleukins (IL-1, IL-10, and IL-6) [3]. Electrochemical impedance spectroscopy was also used to determine TNF- $\alpha$ and IL-12 
with limits of detection of 60 and $3.4 \mathrm{pg} / \mathrm{mL}$, respectively, by conjugation of antibodies to gold nanoparticles through self-assembled monolayers of 16-mercaptohexadecanoic acid [20]. It is worth to mention that commercial ELISA kits for the single determination of IL-1 $\beta$ or TNF- $\alpha$ are available but they are characterized by the long assay times required and the relatively large sample volumes.

The immunosensor described in this work for the simultaneous determination of IL- $\beta 1$ and TNF- $\alpha$ involves the use of dual screen-printed carbon electrodes (SPCEs) modified with 4-carboxyphenyl-functionalized double-walled carbon nanotubes (HOOC-Phe-DWCNTs) as electrochemical scaffolds for immobilization of the specific anti-IL- $1 \beta$ and anti-TNF- $\alpha$ antibodies. As it is known, that DWCNTs possess several beneficial properties with respect to MWCNTs for the preparation of electrochemical biosensors such as improved lifetimes and high stability under chemical, mechanical and thermal treatments [31]. Moreover, DWCNTs provide better electrochemical behavior than SWCNTs enhancing electron transfer and allowing significant overpotential reduction for various species. Furthermore, they show a higher reactivity, probably as a consequence of the larger number of lattice defects in DWCNTs [32]. Furthermore, upon chemical modification of DWCNTs, the outer cylinder acts as a protective sheath that preserves the electronic properties of the inner tube [41]. Therefore, covalent sidewall chemistry can be performed onto DWCNTs without loss of the intrinsic properties [33].

Capture antibodies were immobilized onto HOOC-Phe-DWCNTs/SPCEs in an oriented form making using the commercial polymeric coating Mix\&Go ${ }^{\mathrm{TM}}$. This polymer contains several metallic complexes with a high efficiency to bind proteins [34]. Sandwich type immunoassays with signal amplification through the use of polyHRP-streptavidin conjugates were performed for each cytokine. This simple and 
relatively low cost immunosensor configuration allowed the development of a sensitive method for the simultaneous determination of IL- $\beta 1$ and TNF- $\alpha$ exhibiting improved overall analytical performance with respect to previously reported data. In addition and in order to demonstrate the analytical usefulness of the developed methodology, the dual immunosensor was applied to the determination of both proteins in human serum and saliva.

\section{Experimental}

\subsection{Reagents and solutions}

Mouse TNF $\alpha$ capture antibody (anti-TNF), biotinylated goat TNF $\alpha$ detection antibody (biotin-anti-TNF), mouse IL1 $\beta$ capture antibody (anti-IL), and biotinylated goat IL $\beta 1$ detection antibody (biotin-anti-IL) were used. These immunoreagents as well as human TNF $\alpha$ and IL $\beta 1$ proteins were those included in the DuoSet ${ }^{\circledR}$ ELISA Development Systems DY201-05 (IL $\beta 1)$, and DY210-05 (TNF $\alpha$ ) from R\&D Systems. Solutions of the respective capture antibodies at a concentration level of $240 \mu \mathrm{g} / \mathrm{mL}$ were prepared by dissolving the product in $500 \mu \mathrm{L}$ PBS. Working solutions were prepared by dilution into a $25 \mathrm{mM}$ buffer solution of $2-(\mathrm{N}$-morpholino) ethanesulfonic acid (MES, Gerbu) of $\mathrm{pH}$ 5.0. Solutions of the respective detection antibodies at 18 $\mu \mathrm{g} / \mathrm{mL}(\mathrm{TNF} \alpha$ ) or $12 \mu \mathrm{g} / \mathrm{mL}$ (IL $\beta 1$ ) concentrations were prepared by dissolving the product in $1 \mathrm{~mL}$ PBS and 1\% BSA. Phosphate buffer saline solution (PBS) consisted of a $137 \mathrm{mM} \mathrm{NaCl}, 2.7 \mathrm{mM} \mathrm{KCl}, 8.1 \mathrm{mM} \mathrm{Na}_{2} \mathrm{HPO}_{4}$, and $1.5 \mathrm{mM} \mathrm{KH}_{2} \mathrm{PO}_{4}$ solution of $\mathrm{pH}$ 7.4 .

Streptavidin labeled with horseradish peroxidase (HRP-Strept, Roche), polyHRP-Strept (85-R200) conjugate (Fitzerald), poly-HRP-Strept (S2438) conjugate (Sigma), and HRP-Strept-AuNPs (Nanovex Biotechnologies) were tested. Solutions 
from each conjugate at the working concentration were prepared in PBS. Double-walled carbon nanotubes functionalized with $p$-aminobenzoic acid (HOOC-Phe-DWCNTs) were provided by Instituto de Nanociencia, Nanotecnología y Materiales Moleculares (INAMOL) de la Universidad de Castilla La Mancha (Toledo), Mix \&Go ${ }^{\mathrm{TM}}$ polymer was from Anteo Diagnostics. Hydroquinone (HQ) and hydrogen peroxide (30\%, w/v) were from Sigma. Bovine serum albumin (BSA) was from GERBU Biotechnik, GmbH. Blocker $^{\circledR}$ Casein in PBS was from Thermo Fisher. Adiponectin (APN, Abcam), ceruloplasmin ( $\mathrm{Cp}, \mathrm{Abcam})$, ghrelin (GHRL, Anaspec) bovine serum albumin (BSA, Gerbu), cholesterol (Chol, Sigma), transforming growth factor $\beta 1$ (TGF $\beta 1$, R\&D Systems), hemoglobin (Hb, Sigma), C-reactive protein (CRP, Fitzgerald), interleukin 6 (IL-6, Abcam), interleukin 8 (IL-8, Abcam), bilirubin (BIL, Aldrich), and leptin (Lep, R\&D Systems) were tested as potential interfering compounds. Deionized water was from a Millipore Milli-Q purification system $\left(18.2 \mathrm{M} \Omega \mathrm{cm}\right.$ at $\left.25^{\circ} \mathrm{C}\right)$.

\subsection{Apparatus and electrodes}

All electrochemical measurements were carried out with a potenciostat from INBEA Biosensores S.L. equipped with the IbGraph software. Screen-printed carbon electrodes (SPCEs, 110 DRP, $\phi 4 \mathrm{~mm}$ ), and dual SPCEs (C 1110 DRP) consisted of two elliptic carbon working electrodes with surface area of $5.6 \mathrm{~mm}^{2}$, were from DropSens (Oviedo, Spain). These electrodes include a carbon counter electrode and a silver pseudo-reference electrode. An Elmasonic S-60 (Elma) ultrasonic bath, a Gyrozen 416 centrifuge (Controltechnica Instruments), and a Vortex homogenizator (Velp Scientifica) were also used. pH measurements were performed using a Crison Basic 20+ $\mathrm{pH}-$ meter. All experiments were performed at room temperature.

\subsection{Samples}


The samples analyzed were lyophilized human serum (S2257, Sigma) spiked with $2.5,5$ and $7.5 \mathrm{pg} / \mathrm{mL}$ IL- $1 \beta$ and 5, 10 and $15 \mathrm{pg} / \mathrm{mL}$ TNF- $\alpha$, and saliva samples obtained from two lab researcher (male smoker and female nonsmoker volunteers) and collected using a Salivette ${ }^{\circledR}$ collection device (Sarstedt).

\subsection{Procedures}

\subsubsection{Preparation of the immunosensors}

A homogeneous dispersion of HOOC-Phe-DWCNTs was prepared by suspending $1 \mathrm{mg}$ of the product in $2 \mathrm{~mL}$ distilled water, addition of $27.4 \mu \mathrm{L}$ of Tween ${ }^{\circledR}$ 20, and ultrasonic stirring for 60 minutes. Thereafter, modification of the electrodes was made by casting $0.5 \mu \mathrm{L}$ of the HOOC-Phe-DWCNTs dispersion onto the electrode surface and allowing drying under IR radiation. This procedure was repeated until a total dispersion volume of $1.5 \mu \mathrm{L}$ was deposited. Next, $2.5 \mu \mathrm{L}$ of Mix\&Go solution were added and incubated for $60 \mathrm{~min}$. Thereafter, the modified electrode was rinsed with water and allowed drying. This washing was accomplished after each electrode modification once incubation was made. Then, the corresponding capture antibody (anti-IL or anti-TNF) was immobilized by casting $2.5 \mu \mathrm{L}$ of $4 \mu \mathrm{g} / \mathrm{mL}$ solution of each antibody and incubating for 60 min. Blocking with $2.5 \mu \mathrm{L}$ of a $2 \%$ BSA solution in PBS for 30 min was then made to minimize unspecific interactions. Thereafter, $2.5 \mu \mathrm{L}$ of the antigen (IL- $\beta 1$ and/or TNF- $\alpha$ ) standard solution or the sample were added and incubated for $60 \mathrm{~min}$. Next, $2.5 \mu \mathrm{L}$ of $0.5 \mu \mathrm{g} / \mathrm{mL}$ biotinylated antibody solution (Biotin-anti-IL or Biotin-anti-TNF) were added and incubated for $45 \mathrm{~min}$. Finally, $2.5 \mu \mathrm{L}$ of a 1/500 diluted poly-HRP-Strept solution were dropped on the modified electrode allowing incubation for $15 \mathrm{~min}$.

\subsubsection{Simultaneous determination of $I L-\beta 1$ and $T N F-\alpha$}


The determination of both cytokines was accomplished by dropping $45 \mu \mathrm{L}$ of a 1 $\mathrm{mM}$ HQ solution onto the surfaces of the dual SPCE immunosensor horizontally positioned and applying a detection potential of $-0.20 \mathrm{~V}$. Once the background current

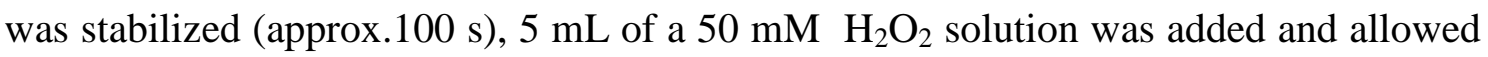
standing for $200 \mathrm{~s}$. The steady state current corresponding to the electrochemical reduction of benzoquinone was used as the analytical readout.

\subsubsection{Samples analysis}

Lyophilized serum was firstly reconstituted by dissolving $370 \mathrm{mg}$ in $5 \mathrm{~mL}$ of deionized water mixing with gentle stirring. Then, the serum was spiked with $2.5,5$ or $7.5 \mathrm{pg} / \mathrm{mL}$ IL- $1 \beta$ and 5,10 or $15 \mathrm{pg} / \mathrm{mL} \mathrm{TNF}-\alpha$, and the determination of both proteins was performed by application of the procedures 2.4.1 and 2.4.2. and interpolation of the amperometric responses in the respective calibration plot constructed with standard solutions.

Regarding saliva, samples were collected from the volunteers after rinsing the mouth thoroughly with water and chewing a cotton swab for $5 \mathrm{~min}$. Then, the swap saturated with saliva was inserted into a vial, sealed with the cap and centrifuged for 10 $\min$ at $3000 \mathrm{x}$ g. The determination was performed immediately by applying the procedure described above using $5-\mu \mathrm{L}$ aliquots of the undiluted sample.

\section{Results and discussion}

The methodologies employed in this work involved the immobilization of capture antibodies onto SPCEs modified with 4-carboxyphenyl-functionalized doublewalled carbon nanotubes (HOOC-Phe-DWCNTs) and application of sandwich type immunoassays for each cytokine with signal amplification through the use of polyHRP-streptavidin conjugates. These strategies took advantage of: i) the well-known 
enhancement of electron transfer processes occurring on DWCNTs-modified electrode surfaces, ii) the high availability of surface confined carboxyl groups onto HOOC-PheDWCNTs, iii) the oriented immobilization of antibodies by means of Mix\&Go polymer, and iv) the amplification of the electrochemical responses with poly-HRP-Strept.

The protocols used for the preparation of the dual immunosensor are described in the Experimental section and schematically displayed in Figure 1. Once Mix\&Go was dropped onto each surface of HOOC-Phe-DWCNTs-modified dual SPCE, anti-IL and anti-TNF antibodies were immobilized and a subsequent blocking step of the remaining active free sites on the electrode surfaces was carried out. Then, sandwich type assays involving the target cytokines and biotinylated detector antibodies were accomplished. A further conjugation with poly-HRP-Strept allowed the amperometric determination of IL-1 $\beta$ and TNF- $\alpha$ to be performed using $\mathrm{H}_{2} \mathrm{O}_{2}$ as HRP substrate and hydroquinone (HQ) as the redox mediator.

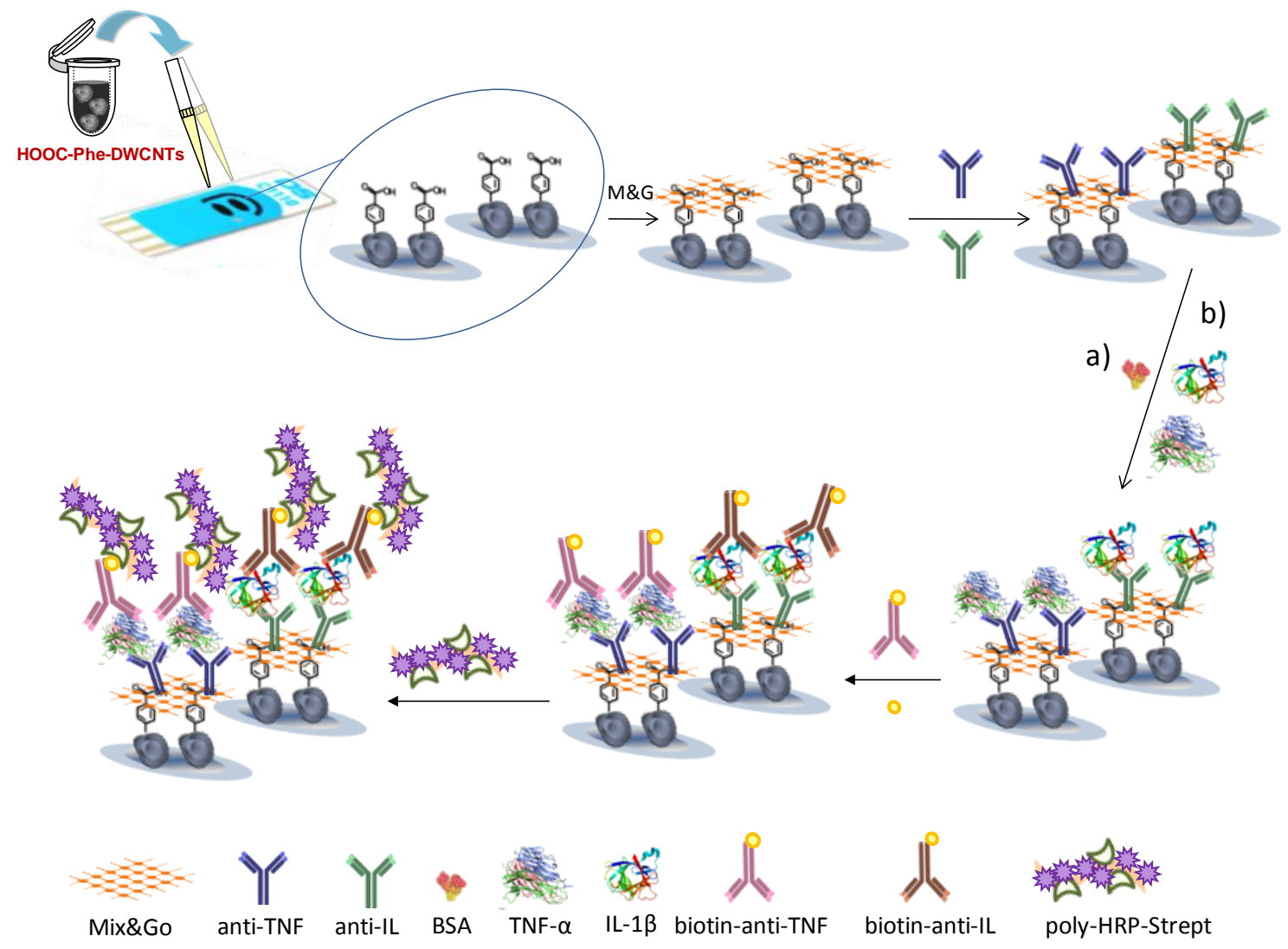


Figure 1. Schematic display of the different steps involved in the preparation of the dual electrochemical immunosensor for multiplexed determination of IL-1 $\beta$ and TNF- $\alpha$ cytokines.

\subsection{Optimization of the experimental variables involved in the preparation of the} dual immunosensor

Variables involved in the preparation of the dual immunosensor for the simultaneous determination of IL- $1 \beta$ and TNF- $\alpha$ were optimized. These studies were performed individually for each cytokine using single modified-SPCEs and included: a) the antibody (anti-IL or anti-TNF) loading onto HOOC-Phe-DWCNTs/SPCE and the time for incubation; b) the Biotin-antibody loading onto IL-1 $\beta$-anti-IL-Phe-DWCNTs/ SPCE or TNF- $\alpha$-anti-TNF-Phe-DWCNTs/SPCE and the time for incubation; c) the blocking step, and d) the type of enzyme label and the loading of poly-HRP-Strept onto Biotin-anti-IL-IL1 $\beta$-anti-IL-Phe-DWCNTs/SPCE or Biotin-anti-TNF-TNF- $\alpha$-anti-TNFPhe-DWCNTs/SPCE. Other variables involved in the preparation of the modified electrodes such as the loading of HOOC-Phe-DWCNTs onto SPCE and the loading of Mix\&Go onto HOOC-Phe-DWCNTs/SPCE, as well as those implied in the electrochemical detection such as the detection potential value or the concentration of $\mathrm{H}_{2} \mathrm{O}_{2}$ and $\mathrm{HQ}$ were the same than those employed in previous papers $[35,3645,46]$. Details on these optimization studies are provided in Supplementary material and Figures S1-S4 and summarized in Table 2.

\subsection{Analytical characteristics of the dual immunosensor}

The calibration plots for IL-1 $\beta$ and TNF- $\alpha$ constructed with the dual immunosensor under the optimized working conditions are shown in Figure 2. Error 
bars were calculated from the measurements carried out with three different dual immunosensors for each concentration. The steady state current vs. IL-1 $1 \beta$ concentration (Figure 2a) followed the adjusted equation $\mathrm{I}, \mathrm{nA}=(624 \pm 15) \log \mathrm{C}, \mathrm{pg} / \mathrm{mL}+393 \pm 18$ $\left(r^{2}=0.996\right)$, with a range of linearity extending between 0.5 and $100 \mathrm{pg} / \mathrm{mL}$ IL- $1 \beta$. Figure $2 \mathrm{~b}$ displays the calibration plot for TNF- $\alpha$ with an equation $\mathrm{I}, \mathrm{nA}=(746 \pm 16)$ $\log \mathrm{C}, \mathrm{pg} / \mathrm{mL}+(245 \pm 22)\left(\mathrm{r}^{2}=0.999\right)$, with a range of linearity from 1 to $200 \mathrm{pg} / \mathrm{mL}$. It is worth to mention that these ranges, covering two orders of magnitude, are adequate for the determination of the cytokines in clinical samples taking into account the expected concentrations at the level of few $\mathrm{pg} / \mathrm{mL}$ [19]. The achieved limits of detection were $0.38 \mathrm{pg} / \mathrm{mL}$ (IL-1 $\beta$ ) and $0.85 \mathrm{pg} / \mathrm{mL}(\mathrm{TNF}-\alpha)$ and were calculated by using the $3 \mathrm{~s}_{\mathrm{b}}$ criterion, where $s_{b}$ was estimated as the standard deviation expressed in concentration units $(\mathrm{n}=10)$ of the measured currents for the blank $(0 \mathrm{pg} / \mathrm{mL}$ IL- $1 \beta$ or TNF- $\alpha)$.

a

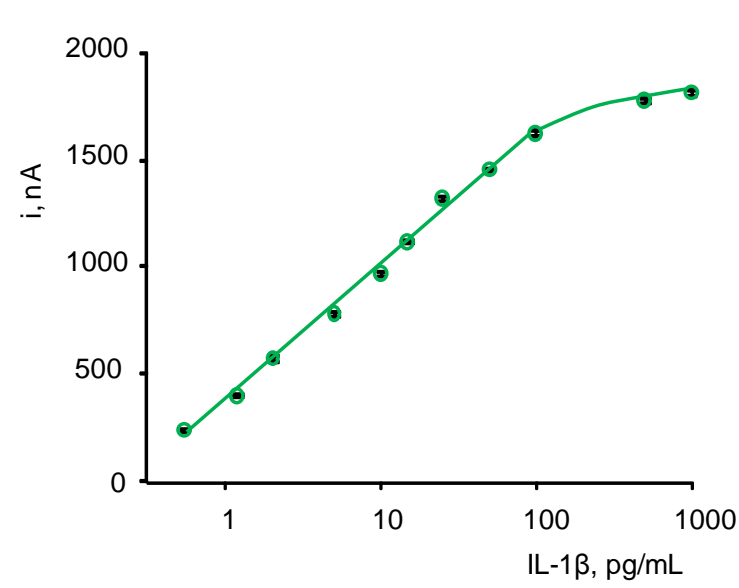

b

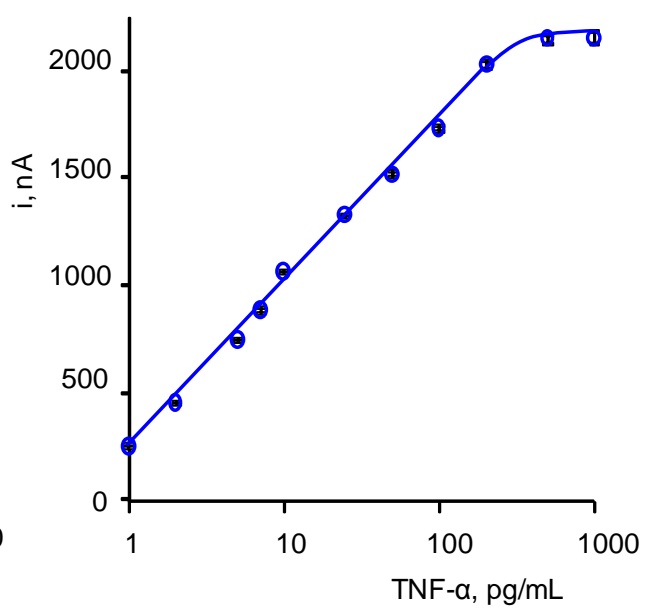

Figure 2. Calibration plots for IL-1 $\beta$ (green) and TNF- $\alpha$ (blue) obtained with the dual poly-HRP-Strept-Biotin-anti-IL-IL1 $\beta$-anti-IL-Phe-DWCNTs/SPCE and poly-HRPStrept-Biotin-anti-TNF-TNF- $\alpha$-anti-TNF-Phe-DWCNTs/SPCE immunosensor. See text for other conditions. 
When these analytical characteristics are compared with those reported for other electrochemical immunosensors (Table 1), it can be concluded that the developed methodology can be ranked among the most sensitive exhibiting low detection limits and linear ranges covering low concentration ranges. It should also be remarked that this excellent analytical performance is accompanied by a relatively short time of analysis. In fact, the whole experimental procedure, once the capture antibodies are immobilized, took $2 \mathrm{~h} 30 \mathrm{~min}$. This period is much shorter than that required when commercial ELISA kits for IL- $1 \beta$ or TNF- $\alpha$ are employed. For example, the assays with the DuoSet ${ }^{\circledR}$ ELISA from R\&D Systems [www.rndsystems.com] last 4h 40 min.

The reproducibility of the measurements was tested with different dual immunosensors prepared on the same day and on different days, and in the absence or in the presence of $50 \mathrm{pg} / \mathrm{mL}$ IL- $1 \beta$ or $100 \mathrm{pg} / \mathrm{mL} \mathrm{TNF-} \alpha$. The relative standard deviation (RSD) values obtained were 0.6 and $2.7 \%$ (IL-1 $\beta)(n=4)$, and 0.3 and $0.7 \%$ $(\mathrm{TNF}-\alpha)(\mathrm{n}=5)$, respectively, for the assays performed on the same day, and $0.8 \%$ and $3.7 \%(\operatorname{IL}-1 \beta) \quad(\mathrm{n}=4)$, and 0.5 and $0.7 \% \quad(\mathrm{TNF}-\alpha) \quad(\mathrm{n}=5)$ respectively, for the measurements made on different days. These results indicated the good level of precision achieved in the fabrication and functioning of the developed dual electrochemical immunosensing platform.

In addition, the storage stability of the anti-IL-Phe-DWCNTs/SPCE and antiTNF-Phe-DWCNTs/SPCE conjugates, once they were blocked with $2 \%$ BSA, was tested under storage at $-20{ }^{\circ} \mathrm{C}$ in a dry environment. Several bioconjugates were prepared on the same day, stored, and used to construct on different days, poly-HRPStrept - Biotin - anti - IL - IL1 $\beta$ - anti - IL - Phe - DWCNTs / SPCE and poly - HRPStrept-Biotin-anti-TNF-TNF- $\alpha$-anti-TNF-Phe-DWCNTs/SPCE dual immunosensors. The amperometric currents measured for $50 \mathrm{pg} / \mathrm{mL}$ IL-1 $\beta$ and $100 \mathrm{pg} / \mathrm{mL}$ TNF- $\alpha$ 
solutions (Figure S5) remained within the control limits set by \pm 3 times the standard deviation of the measurements $(n=3)$ carried out on the first day, during at least 40 days. This excellent storage stability suggests the possibility of preparing a set of anti-IL-PheDWCNTs/SPCE and anti-TNF-Phe-DWCNTs/SPCE conjugates and storing them under the above mentioned conditions until their use for the preparation of the immunosensors was requested.

\subsection{Selectivity studies}

The effect of potential interfering compounds that may be present together with the target cytokines in biological samples on the electrochemical responses obtained with the dual immunosensors was studied. As the used antibodies exhibit demonstrated specificity for IL- $1 \beta$ or TNF- $\alpha$ against other cytokines such as IL- $1 \alpha$, or TNF- $\beta$, TNF-RI and TNF-TII, respectively, the compounds tested were: adiponectin (APN), bovine serum albumin (BSA), cholesterol (Chol), C-reactive protein (CRP), hemoglobin (Hb), interleukin 6 (IL-6), interleukin-8 (IL-8), transforming growth factor $\beta 1$ (TGF- $\beta 1$ ), bilirubin (BIL), ceruloplasmin (Cp), ghrelin (GHRL) and leptin (LEP). Figure 3 shows a comparison of the amperometric responses obtained with the dual immunosensor for 0 and $10 \mathrm{pg} / \mathrm{mL}$ IL- $1 \beta$ or TNF- $\alpha$ in absence and in the presence of each tested compound at their normal concentration levels in human serum. As it is clearly shows, no significant effect on the dual electrochemical immunosensor response was apparent in the presence of any non-target compound, thus demonstrating the excellent selectivity of the proposed configuration for simultaneous IL- $1 \beta$ and TNF- $\alpha$ determination. 

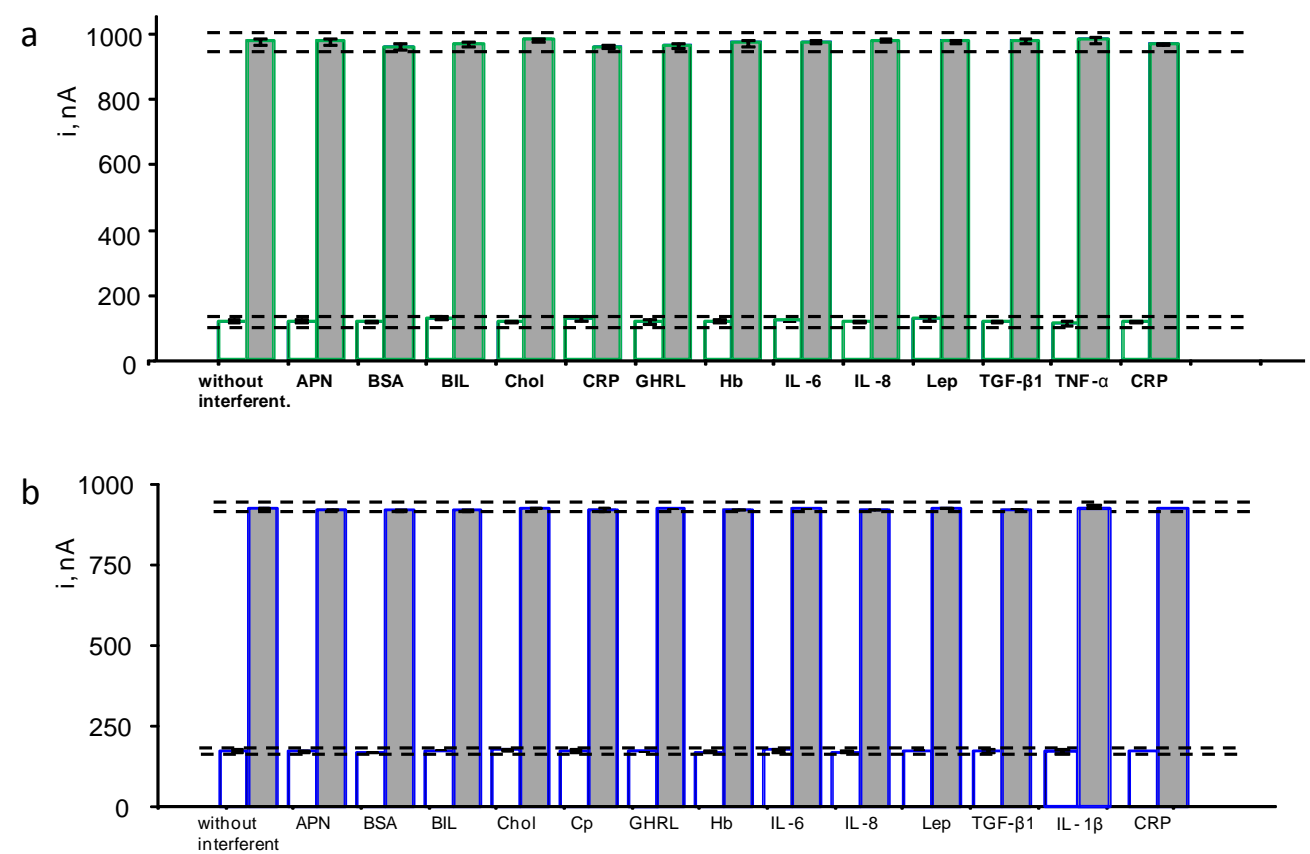

Figure 3. Effect of the presence of $20 \mu \mathrm{g} / \mathrm{mL}$ APN, $5 \mathrm{mg} / \mathrm{mL} \mathrm{BSA}, 190 \mathrm{ng} / \mathrm{mL}$ bilirubin (BIL), $20 \mu \mathrm{g} / \mathrm{mL}$ Chol, $500 \mu \mathrm{g} / \mathrm{mL}$ ceruloplasmin (Cp), $500 \mathrm{pg} / \mathrm{mL}$ ghrelin (GHRL), 50 $\mu \mathrm{g} / \mathrm{mL} \mathrm{Hb}, 50$ pg/mL IL-6, 30 pg/mL IL-8, 5 ng/mL leptin (Lep), 500 pg/mL TGF$\beta 1,100 \mathrm{pg} / \mathrm{mL}$ TNF- $\alpha$ (a) or $50 \mathrm{pg} / \mathrm{mL}$ IL-1 $\beta$ (b), $1 \mu \mathrm{g} / \mathrm{mL}$ CRP on the amperometric responses obtained for 0 (white) and 10 (grey) pg/mL IL-1 $\beta$ (a) or TNF- $\alpha$ (b) at the dual electrochemical immunosensor. See the text for more information.

Moreover, the possible cross-talking between the adjacent working modified SPCEs in the dual configuration and reaction products was evaluated by comparing the amperometric responses recorded at the poly-HRP-Strept-Biotin-anti-IL-IL1 $\beta$-anti-ILPhe-DWCNTs/SPCE where $10 \mathrm{pg} / \mathrm{mL}$ TNF- $\alpha$ were deposited, and at the poly-HRPStrept-Biotin-anti-TNF-TNF- $\alpha$-anti-TNF-Phe-DWCNTs/SPCE with $25 \mathrm{pg} / \mathrm{mL}$ IL- $1 \beta$, with those obtained for 0 or $25 \mathrm{pg} / \mathrm{mL} \mathrm{IL-} \beta 1$ and $10 \mathrm{pg} / \mathrm{mL}$ TNF- $\alpha$, respectively. Figure 4 shows clearly that no significant differences in the amperometric responses of immunosensors exist in the absence of the non-target antigen, thus demonstrating that no appreciable cross-talking occurred with the developed dual immunosensor. 

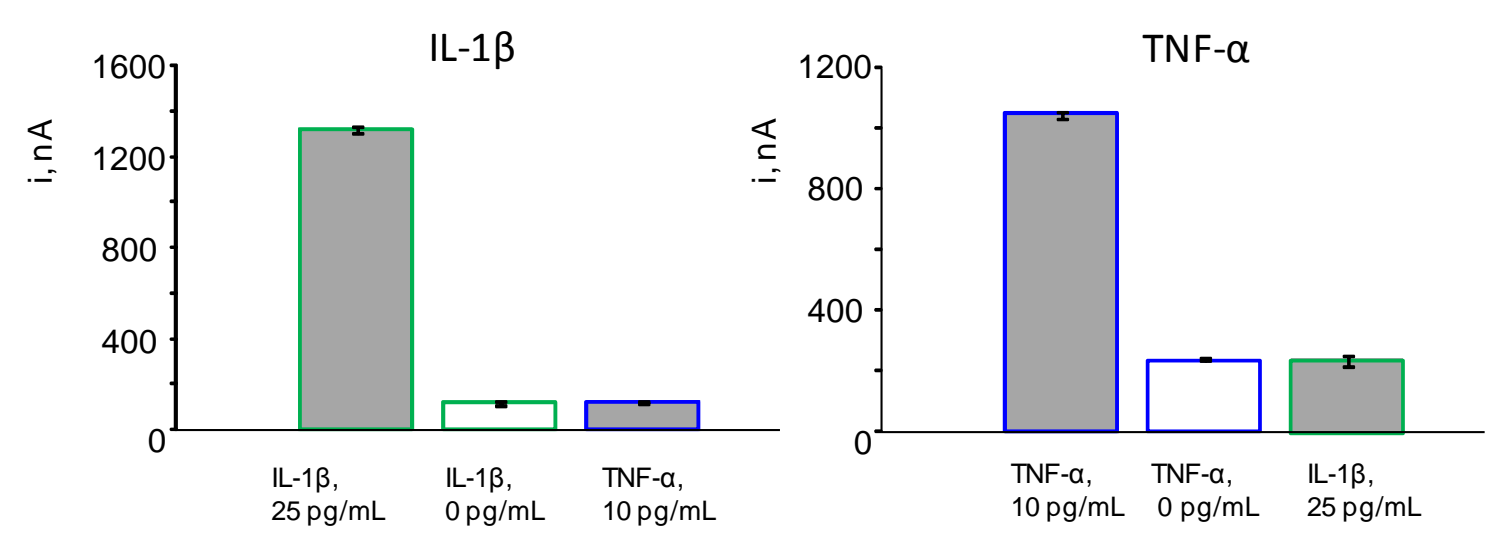

Figure 4. Amperometric responses recorded with the dual immunosensor at poly-HRPStrept-Biotin-anti-IL-IL1 $\beta$-anti-IL-Phe-DWCNTs/SPCE for 25 or $0 \mathrm{pg} / \mathrm{mL}$ IL-1 $\beta$ (green), or $10 \mathrm{pg} / \mathrm{mL}$ TNF- $\alpha$ (blue) (a), and at poly-HRP-Strept-Biotin-anti-TNF-TNF$\alpha$-anti-TNF-Phe-DWCNTs/SPCE for with 10 or $0 \mathrm{pg} / \mathrm{mL}$ TNF- $\alpha$ (blue), or $25 \mathrm{pg} / \mathrm{mL}$ IL-1 $\beta$ (green) (b). See the text for more information.

\subsection{Simultaneous determination of IL-1 $\beta$ and TNF- $\alpha$ in spiked serum and saliva}

The developed dual immunosensor was applied to the simultaneous determination of IL-1 $\beta$ and TGF- $\beta$ in human serum spiked at clinically relevant concentration levels $[37,38]$ and in real saliva samples. Regarding serum, the possible existence of matrix effects was initially evaluated by constructing a calibration plot in the sample (lyophilized serum from Sigma) which was spiked with IL-1 $\beta$ over the 2.5 to $7.5 \mathrm{pg} / \mathrm{mL}$, and with TNF- $\alpha$ over the 5 to $15 \mathrm{pg} / \mathrm{mL}$ concentration ranges, respectively. The equations obtained for the respective calibration graphs were $\mathrm{I}, \mathrm{nA}=(597 \pm 6) \log$ $\mathrm{C}, \mathrm{pg} / \mathrm{mL}+(400 \pm 4),\left(\mathrm{r}^{2}=0.9998\right)$, and $\mathrm{I}, \mathrm{nA}=(766.3 \pm 0.2) \log \mathrm{C}, \mathrm{pg} / \mathrm{mL}+(237.8 \pm$ $0.2),\left(r^{2}=0.9999\right)$ for IL-1 $\beta$ and TNF- $\alpha$, respectively. The comparison of the slope values with those calculated for the calibration plots constructed with standard solutions by 
applying the Student's t test showed texp values 1.655 (IL-1 $\beta$ ) and 1.231 (TNF- $\alpha$ ) lower than that tabulated $\mathrm{t}, 2.306$, therefore indicating that no statistically significant differences existed between the slopes values for both types of calibration plots. Therefore, the determination of IL- $1 \beta$ and TNF- $\alpha$ could be carried out by interpolation of the current values measured for the serum samples into the calibration plots constructed with the standard solutions. Table 3 summarizes the results obtained by triplicate analysis of samples spiked at three different concentration levels: 2.5, 5.0 and $7.5 \mathrm{pg} / \mathrm{mL}$ IL- $1 \beta$ and 5.10 and $15 \mathrm{pg} / \mathrm{mL}$ TNF- $\alpha$. As it can be seen, recoveries ranged between 98 and $104 \%$, indicating the reliability of the approach to determine low IL$1 \beta$ and TNF- $\alpha$ concentrations in serum following a simple working protocol.

Furthermore, two different saliva samples corresponding to a male smoker and a female nonsmoker volunteers were analyzed to determine the endogenous content of both IL-1 $\beta$ and TNF- $\alpha$. Considering the complexity of saliva, we also evaluated the potential occurrence of matrix effect. A calibration plot was constructed by adding known cytokine concentrations between 0.5 and $50 \mathrm{pg} / \mathrm{mL}$ for IL- $1 \beta$ and 2 and 40 $\mathrm{pg} / \mathrm{mL}$ for TNF- $\alpha$ to saliva from both patients. The respective slope values of the linear ranges $(630 \pm 14$ and $627 \pm 5$ for IL- $1 \beta ; 745 \pm 37$ and $758 \pm 5$ for TNF- $\alpha)$ were statistically compared with those calculated from the calibration graphs with IL-1 $\beta$ or TNF- $\alpha$ standards. (624 \pm 15 and $746 \pm 16 \mathrm{nA}$ per decade of concentration $)$. No significant difference for a $95 \%$ confidence interval $\left(t_{\exp }=0.27\right.$ and 0.17 (IL-1 $\left.\beta\right)$ and 0.10 and $0.67(\mathrm{TNF}-\alpha)$ were lower than $\left.\mathrm{t}_{\mathrm{tab}}=2.201\right)$. Accordingly, the determination of IL-1 $\beta$ and TNF- $\alpha$ in saliva was accomplished by interpolation of the measured current in the samples into the calibration plot constructed with standard solutions. Table 4 summarizes the results obtained in the analysis of saliva. Interestingly, the found values re consistent with those reported using other approaches [49]-and, as expected, no 
significant differences in salivary concentrations of the cytokines are apparent between smoker and non smoker individuals.

In addition, the results were compared with those obtained using the DuoSet ${ }^{\circledR}$ ELISA Development Systems DY201-05 (IL1 $\beta$ ), and DY210-05 (TNF $\alpha$ ) from R\&D Systems. The ELISA protocols were similar for both cytokines and, briefly, involved incubating the capture antibody solution into the wells overnight at room temperature, followed by washing and blocking with BSA for a minimum of $1 \mathrm{~h}$. Then, $100 \mu \mathrm{L}$ of the sample or standards per well were added and incubated $2 \mathrm{~h}$ at room temperature. After washing, $100 \mu \mathrm{L}$ of the detection antibody (biotinylated antibody) were added to each well and incubated $2 \mathrm{~h}$ at room temperature. Once washed, $100 \mu \mathrm{L}$ of the HRP-Strept solution were added to each well leaving for $20 \mathrm{~min}$ at room temperature, and color development was achieved by the addition of TMB substrate solution. Finally, after 20 min, an acid "stop solution" was added, and the absorbance was mesured at $450 \mathrm{nM}$. As it can be deduced from Table 4, the results obtained are consistent with those provided by the immunosensor. Indeed, the concentration values found by both methods were statistically compared by means of the Student's $t$ test. The $t_{\exp }$ values, 0.30 and 0.15 for IL- $1 \beta$ and 0.55 and 0.30 for TNF- $\alpha$, were lower than the tabulated t value (2.16) and, therefore, no significant differences were found between both methodologies at a significance level of 0.05 . These results demonstrate clearly the usefulness of the developed immunosensor to determine simultaneously IL-1 $\beta$ and TNF- $\alpha$ at clinically relevant concentrations in biological fluids such as serum or saliva.

\section{Conclusions}

An electrochemical dual platform for the simultaneous determination of IL- $\beta 1$ and TNF- $\alpha$ cytokines is reported in this work. The platform profits the benefits inherent to electrode modification with 4-carboxyphenyl-functionalized double-walled carbon 
nanotubes where specific anti-IL-1 $\beta$ and anti-TNF- $\alpha$ antibodies were immobilized in an oriented manner using the commercial polymeric coating Mix $\& \mathrm{Go}^{\mathrm{TM}}$. Sandwich type immunoassays with signal amplification by means of poly-HRP-streptavidin conjugates allowed the development of a sensitive method for the simultaneous determination of IL- $\beta 1$ and TNF- $\alpha$ with improved analytical performance with respect to previously reported approaches and commercial ELISA kits. The dual immunosensor was successfully applied to the determination of both cytokines at clinically relevant concentrations in biological fluids such as serum or saliva. In this context, an important point to be remarked is the simultaneous assay takes about $2 \mathrm{~h} 30 \mathrm{~min}$ once the capture antibodies were immobilized. Conversely, ELISAs require $4 \mathrm{~h} 40 \mathrm{~min}$ for each determination. Moreover, the reagents consumption is much lower in the method using the dual immunosensor since it only requires $2.5 \mu \mathrm{L}$ each time while $100 \mu \mathrm{L}$ of the same reagents are needed in the ELISA protocol.

\section{Acknowledgments}

Financial support of Spanish Ministerio de Economía y Competitividad, Research Projects CTQ2015-70023-R, and CTQ2015-71955-REDT, and NANOAVANSENS Program from Comunidad de Madrid (S2013/MT-3029), is gratefully acknowledged.

\section{References}

[1] G. Liu, M. Qi, M.R. Hutchinson, G.Yang, E.M. Goldys, Recent advances in cytokine detection by immunosensing, Biosens. Bioelectron.79 (2016) 810-821.

[2] J. Whicher, S. Evans, Cytokines in disease, Clin.Chem.36 (1990) 1269-1281.

[3] A. Baraket, M. Lee, N. Zine, M.G. Trivella, M. Zabala, J. Bausells, M. Sigaud, N. Jaffrezic-Renault, A. Errachid, A fully integrated electrochemical bioMEMS fabrication 
process for cytokine detection: Application for heart failure, Proc. Eng, 87 (2014) 377379.

[4] S.S. Shaftel, W.S.T. Griffin, M.K. O’Banion, The role of interleukin-1 in neuroinflammation and Alzheimer disease: An evolving perspective, J. Neuroinflammation, 5 (2008) 7.

[5] M. Feldmann, F.M. Brennan, R.N. Maini, Role of cytokines in rheumatoid arthritis, Annu. Rev. Immunol. 14 (1996) 397-440.

[6] M.A. Palladino, F. R. Bahjat, E.A. Theodorakis, L.L. Moldawer, Anti-TNF-[alpha] therapies: the next generation. Nat. Rev. Drug Discov. 2 (2003) 736-746.

[7] K. Last-Barney, C.A. Homon, R.B. Faanes, V.J. Merluzzi, Synergistic and overlapping activities of tumor necrosis factor alpha and IL-1. J. 1mmunol, 141 (1988) 527-530. [8] P. Musto, R. Matera, M. M. Minervini, C. Checchia-de Ambrosio, C. Bodenizza, A. Falcone, M. Carotenuto, Low serum levels of tumor necrosis factor and interleukin-1 beta in myelodysplastic syndromes responsive to recombinant erythropoietin, Haematol. 79 (1994) 265-268.

[9] N. Demircan, B.G. Safran, M. Soylu, M.M. Ozcan, S. Sizmaz, Determination of vitreous interleukin-1 (IL-1) and tumour necrosis factor (TNF) levels in proliferative diabetic retinopathy, Eye, 20 (2006) 1366-1369.

[10] A. P. Di Battista, S.G. Rhind, M.G. Hutchison, S. Hassan, M.Y. Shiu, K. Inaba, J. Topolovec-Vranic, A. C. Neto, S.B. Rizoli, A.J. Baker, Inflammatory cytokine and chemokine profiles are associated with patient outcome and the hyperadrenergic state following acute brain injury, J. Neuroinflamm. 13 (2016) 40.

[11] C. E. Krause, B.A. Otieno, G.W. Bishop, G. Phadke, L. Choquette, R.V. Lalla, D.E. Peterson, J.F. Rusling, Ultrasensitive microfluidic array for serum pro- 
inflammatory cytokines and C-reactive protein to assess oral mucositis risk in cancer patients, Anal. Bioanal. Chem., 407 (2015) 7239-7243.

[12] V. Brailo, V. Vucicevic-Boras, J. Lukac, D. Biocina-Lukenda, I. Zilic-Alajbeg, A. Milenovic, M. Balija, Salivary and serum interleukin 1 beta, interleukin 6 and tumor necrosis factor alpha in patients with leukoplakia and oral cancer, Med. Oral Patol. Oral Cir. Bucal 17 (2012) 10-15

[13] F. I. Fernandes Gomes, M. G. Brito Aragão, F. C. Barroso Barbosa, M. Marques Bezerra, V.de P. Teixeira Pinto, H. Vasconcelos Chaves, Inflammatory Cytokines Interleukin-1 $\beta$ and Tumour Necrosis Factor- $\alpha$ - Novel Biomarkers for the Detection of Periodontal Diseases: a Literature Review, J Oral Maxillofac Res., 7 (2016) e2.

[14] L. Yuan, X. Hua, Y. Wu, X. Pan, S. Liu, Polymer-Functionalized Silica Nanosphere Labels for Ultrasensitive Detection of Tumor Necrosis Factor-alpha, Anal. Chem. 83 ( 2011), 83, 6800-6809.

[15] Z. Yin, Y. Liu, L-P. Jiang, J.-J. Zhu, Electrochemical immunosensor of tumor necrosis factor alpha based on alkaline phosphatase functionalized nanospheres, Biosens. Bioelectron. 26 (2011) 1890-1894.

[16] T. Li, Z. Si, L. Hu, H. Qia, M. Yang, Prussian Blue-functionalized ceria nanoparticles as label for ultrasensitive detection of tumor necrosis factor alpha, Sens. Actuators B 171- 172 (2012) 1060-1065.

[17] Y. Hou, T. Li, H. Huang, H. Quan, X. Miao, M. Yang, Electrochemical immunosensor for the detection of tumor necrosis factor alpha based on hydrogel prepared from ferrocene modified amino acid, Sens. Actuators, B. 182 (2013) 605-609. [18] Z. Sun, , L. Deng, H. Gan, R. Shen, M. Yang, Y. Zhang, Sensitive immunosensor for tumor necrosis factor alpha based on dual signal amplification of ferrocene modified 
self-assembled peptide nanowire and glucose oxidase functionalized gold nanorod, Biosens. Bioelectron. 39 (2013) 215-219.

[19] M. Mazloum-Ardakani, L. Hosseinzadeh, Z. Taleat, Two kinds of electrochemical immunoassays for the tumor necrosis factor $\alpha$ in human serum using screen-printed graphite electrodes modified with poly(anthranilic acid), Microchim. Acta, 181 (2014) 917-924.

[20] J. T. La Belle, U. K. Demirok, D.R. Patelc, C. B. Cook, Development of a novel single sensor multiplexed marker assay, Analyst, 136 (2011) 1496- 1501.

[21] F. Bettazzi, L. Enayati, I. Campos Sánchez, R. Motaghed, M. Mascini, I. Palchetti, Electrochemical bioassay for the detection of $\mathrm{TNF}-\mathrm{a}$ using magnetic beads and disposable screen-printed array of electrodes, Bioanalysis 5 (2013) 11-19.

[22] L. Yuan, W. Wei, S.Q. Liu, Label-free electrochemical immunosensors based on surface-initiated atom radical polymerization, Biosens. Bioelectron. 38 (2012) 79-85.

[23] S. Weng, M. Chen, C. Zhao, A. Liu, L. Lin, Q. Liu, J. Lin, X. Lin, Label-free electrochemical immunosensor based on $\mathrm{K}_{3}\left[\mathrm{Fe}(\mathrm{CN})_{6}\right]$ as signal for facile and sensitive determination of tumor necrosis factor-alpha, Sens. Actuators B 184 (2013) 1-7.

[24] T. S. Pui, P. Kongsuphol, S. K. Arya, T. Bansal, Detection of tumor necrosis factor (TNF-alpha) in cell culture medium with label free electrochemical impedance spectroscopy, Sens. Actuators B 181 (2013) 494-500.

[25] U. Eletxigerra, J. Martínez-Perdiguero, S. Merino, R. Villalonga, J.M. Pingarrón, S. Campuzano, Amperometric magnetoimmunoassay for the direct detection of tumor necrosis factor alpha biomarker in human serum Anal. Chim. Acta 838 (2014) 37-44. [26] P. Kongsuphol, H. H. Ng, J. P. Pursey, S. K. Arya, C. C. Wong, E. Stulz, M. K. Park, EIS-based biosensor for ultra-sensitive detection of TNF- $\alpha$ from non-diluted human serum, Biosens. Bioelectron. 61 (2014) 274-279. 
[27] M.Mazloum-Ardakani, L.Hosseinzadeh, Highly-sensitive label-free immunosensor for tumor necrosis factor a based on Ag@Pt core-shell nanoparticles supported on MW CNTs as an efficient electrocatalyst nanocomposite, RSC Adv. 5 (2015) 70781-70786.

[28] M. Mazloum-Ardakani, L.Hosseinzadeh, A. Khoshroo, Label-free electrochemical immunosensor for detection of tumor necrosis factor $\alpha$ based on fullerenefunctionalized carbon nanotubes/ionic liquid, J. Electroanal. Chem. 757 (2015) 58-64.

[29] U. Eletxigerra, J. Martinez-Perdiguero, S. Merino, Disposable microfluidic immuno-biochip for rapid electrochemical detection of tumor necrosis factor alpha biomarker, Sens. Actuators B, 221 (2015) 1406-1411.

[30] G. Baydemir, F. Bettazzi, I. Palchetti, D. Voccia, Strategies for the development of an electrochemical bioassay for TNF- alpha detection by using a non-immunoglobulin bioreceptor, Talanta, 151 (2016) 141-147.

[31] A.A. Green, M.C. Hersam, Properties and application of double-walled carbon nanotubes sorted by outer-wall electronic type, ACS Nano 5 (2011) 1459-1467.

[32] M. Pumera, Electrochemical properties of double wall carbon nanotube electrodes, Nanoscale Res. Lett. 2 (2007) 87-93.

[33] E. Moore, B.S. Flavel, A.V. Ellis, J.G. Shapter, Comparison of double-walled with single-walled carbon nanotube electrodes by electrochemistry, Carbon 49 (2011) 26392647.

[34] H.W. Ooi, S. J. Cooper, C.-Y. Huang, D. Jennins, E. Chung, N. J. Maeji, A.K. Whittaker, Coordination complexes as molecular glue for immobilization of antibodies on cyclic olefin copolymer surfaces, Anal. Biochem. 456 (2014) 6-13.

[35] M. Eguílaz, M. Moreno-Guzmán, S. Campuzano, A. González-Cortés, P. YáñezSedeño, J.M. Pingarrón, An electrochemical immunosensor for testosterone using 
functionalized magnetic beads and screen-printed carbon electrodes, Biosens. Bioelectron. 26 (2010) 517-522

[36] I. Ojeda, M. Moreno-Guzmán, A. González-Cortés, P. Yáñez-Sedeño, J. M. Pingarrón, Electrochemical magnetoimmunosensor for the ultrasensitive determination of interleukin-6 in saliva and urine using poly-HRP streptavidin conjugates as labels for signal amplification, Anal. Bioanal. Chem. 406 (2014) 6363-6371

[37] Y. Dogan, S. Akarsu, B. Ustundag, E. Yilmaz, M.K. Gurgoze, Serum IL-1 $\beta$, IL-2 and IL-6 in insulin-dependent diabetic children, Mediators Inflamm. (2006) 1-6 [38] O. Arica, M. Aral, S. Sasmaz, P. Ciragil, Serum levels of TNF- $\alpha$, IFN- $\gamma$, IL-6, IL-8, IL-12, IL-17 and IL-18 in patients with active psoriasis and correlation with disease severity, Mediators Inflamm (2005) 273-279 